\title{
Tibial bowing in children - what is normal? a radiographic study
}

\author{
Isabella Zbinden $^{1} \cdot$ Erich Rutz $^{2}$ - Jon A. Jacobson ${ }^{3}$ • Olaf Magerkurth ${ }^{4,5}$
}

Received: 13 November 2014 / Revised: 29 March 2015 / Accepted: 8 April 2015 / Published online: 30 May 2015

(C) European Society of Radiology 2015

\begin{abstract}
Purpose To define osseous landmarks on tibia radiographs in order to establish age-related normal values characterizing physiological tibial bowing in children.

Materials and methods Five hundred and twenty-six patients aged 0-17 years with normal radiographs of the lower legs were identified and retrospectively reviewed by two blinded radiologists. In anteroposterior (ap)/lateral (lat)-views, 3 lines defined tibial length and angulation. Line-A connecting proximal to distal corner of tibial metaphysic, lines $\mathrm{B}$ and $\mathrm{C}$ corresponding to corners of tibial metaphysis. Angle $\mathrm{A} / \mathrm{B}$ defines proximal, $\mathrm{A} / \mathrm{C}$ distal tibial-angulation. Tibial curvature is defined by distance of line-D parallel to A and tangential to tibial cortex. Normal values were calculated with linear-regression. Intra-/Interreader agreement were tested with a Bland-Altman-plot.

Results Intrareader-agreement: Reader 1 showed a bias of 0.1 , standard-deviation of bias was 1.9 and $95 \%$-limits-ofagreement -3.9- 3.7. Reader 2: -0.01, 2.4 and -4.7- 4.7. Interreader: $0.2,1.6$ and $-2.9-3.3$. Angle-A/B ap was $80-$
\end{abstract}

Olaf Magerkurth

dr.o.magerkurth@gmail.com

1 Department of Radiology, University of Basel, Petersgraben 4, Basel 4031, Switzerland

2 Department of Orthopedic Surgery, University Children's Hospital, Spitalstrasse 33, Basel 4056, Switzerland

3 Department of Radiology, University of Michigan, 1500 East Medical Center Drive, Ann Arbor, MI 48109-5326, USA

4 Department of Radiology, University Children's Hospital, Spitalstrasse 33, Basel 4056, Switzerland

5 Department of Radiology, Kantonsspital Baden, Im Ergel 1, 5404 Baden, Switzerland $100^{\circ}$, increasing with age (86.5-88); angle-AC ap was 82$107^{\circ}(96.8-90.5)$, angle-AB lat was $81-107^{\circ}(93.0-98.0)$; angle-AC lat was 76-102 (89.5-86.5); depth of curve ap was $0-11 \%(8-3.5)$ and lat 2-13\%, (8.5-3.5).

Conclusion Age dependent tibial bowing can be assessed with this new measurement system and age-related normalvalues characterizing physiological tibial bowing in children is established.

Key Points

- Tibial Bowing is diagnosed on conventional radiographs.

- Existing Methods provide limited level of confidence.

- New methods provide easy to assess landmarks in all patient ages.

- Existing methods require higher radiation dose compared to new method presented.

Keywords Radiograph · Imaging · Tibia - Lower extremity deformities $\cdot$ Pediatric

\section{Introduction}

Physiological bowing of the lower extremities, especially the tibia with varus angulation, can be a normal finding in neonates and infants [1]. Nevertheless, varus angulation is a frequent cause of referrals to paediatric orthopaedic clinics [1]. The role of the physician is to determine whether the bowing is physiologic or pathologic. There are numerous causes for pathologic bowing deformities of the tibia, such as congenital bowing [2, 3], congenital pseudarthrosis [4], Blount disease [1], neurofibromatosis [5], osteogenesis imperfecta [6], rickets [7], campomelic dysplasia [8]), achondroplasia [1], and Cozen fracture [9]. Abnormal lower leg bowing can be treated by bracing $[10,11]$ or osteotomies [12-20] if appropriately diagnosed. 
Lower leg bowing is usually assessed by measurements of the metaphyseal-diaphyseal angle [21] or standardized angles between the anatomical or biomechanical axis to articular surfaces [22]. In our clinical practice we have recognized difficulties with defining the long axis in "bowed" legs and articular surfaces with incomplete epiphyseal ossification. In addition, the metaphyseal-diaphyseal angle does not take into account the location of the deformity. Moreover, in children who cannot stand, weight-bearing images cannot be obtained and measurements may underestimate deformities. Lastly, the above described measurements require imaging of the complete lower extremity and pelvis on radiography, which has a higher radiation dose compared to isolated radiographs of the distal lower extremity.

The purpose of this study is to define osseous landmarks on tibia radiographs to introduce a news measurement system and to establish age-related normal values characterizing the physiological tibial bowing in children.

\section{Materials and methods}

Institutional review board approval was obtained for this retrospective study and informed consent was waived. The departmental radiology information system was searched for all patients who underwent radiography of the distal lower extremity from 2006 to 2012. All imaging and clinical records were also reviewed. Patients with incomplete imaging, fractures, and skeletal deformities were excluded, as well as patients imaged to evaluate for clinically-suspected skeletal deformity. Included were patients who underwent radiography of the tibia after suspected trauma where a fracture initially could be excluded or occult fractures were excluded because patients did not receive follow-up imaging because of complete remission of pain after one week (Fig. 1). Of these, only those were included where clinical history excluded the possibility of skeletal deformity (Fig. 1). Images were retrospectively reviewed by two radiologists (O.M. with 2 years experience in musculoskeletal radiology and 3 years paediatric radiology; I.Z. with 3 years experience in radiology) who were blinded to patient related information on images.

\section{Imaging technique}

All patients underwent radiography of the lower leg and included anteroposterior and lateral views. radiography field-ofview included the distal femur to cover the knee joint and the distal tip of the fibula to include the tibiotalar joint.

\section{Image analysis}

Images were reviewed retrospectively by one fellowshiptrained musculoskeletal and paediatric radiologist (O.M. and one resident in the third year of training (I.Z.) who were blinded to patient age, gender, and date of radiographic examination. Images were reviewed in a randomized order on a picture archiving and communication system workstation
Fig. 1 Examples for patients excluded from the study. Anteroposterior view of right lower extremities of with a clinically confirmed Blount disease with beaking of the medial aspects of the metaphysis (a), clinically confirmed rickets without radiographic changes (b), a non-displaced fracture of the proximal tibia (c) and a bucket handle fracture of the proximal tibia (d)
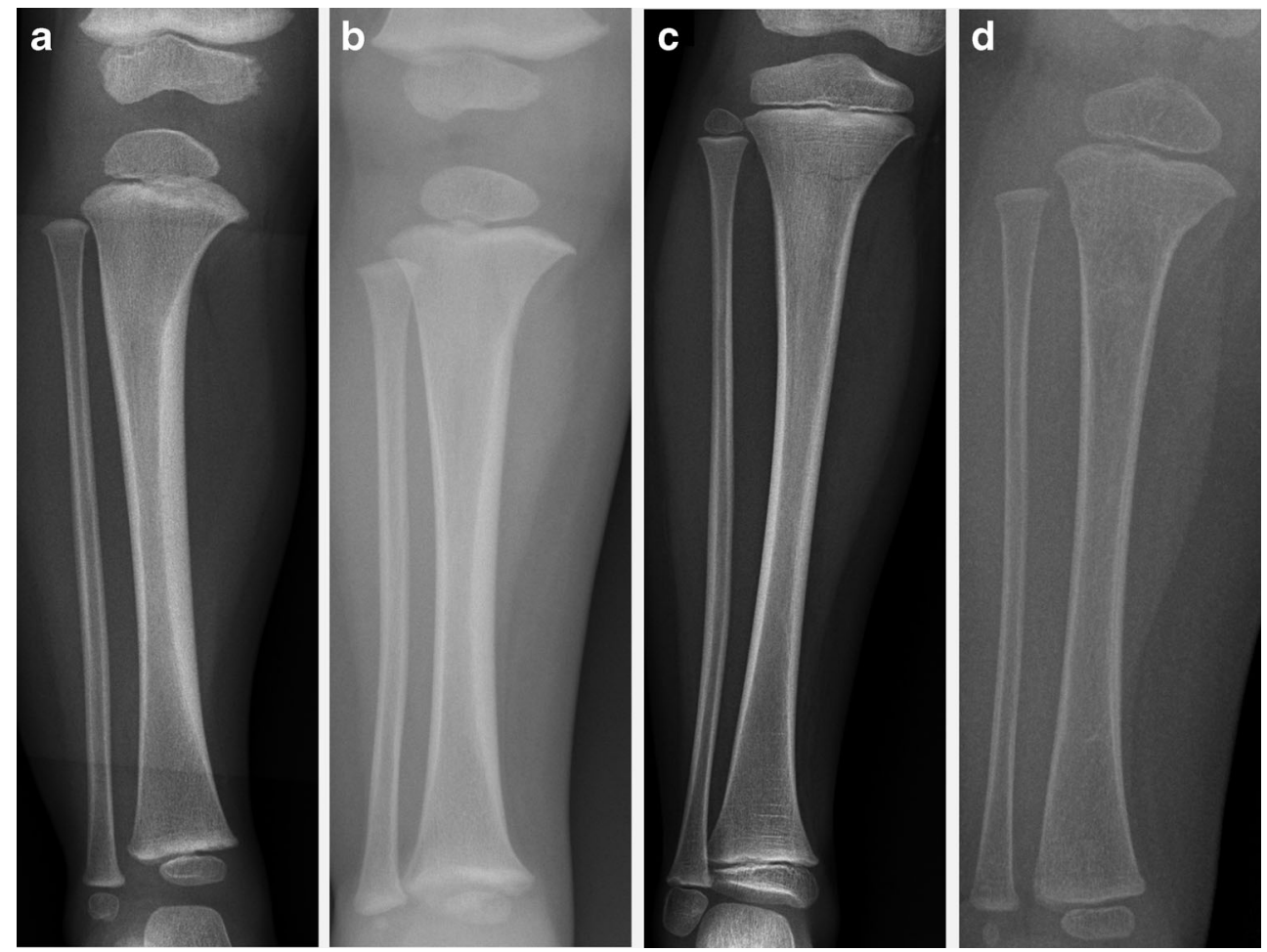
(GE, Healthcare, Barrington, IL, USA) with measurements obtained by using a digital measurement caliper tool.

\section{Measurements: anteroposterior view}

\section{Medial tibial length and angulation}

Three lines were drawn (Fig. 2). The first (Line A) connects the medial aspect of the proximal tibial metaphysis to the medial aspect of the distal metaphysis, which defines the medial tibia length. A second (Line B) connects the corners of the proximal tibial metaphysis, while a third line (Line C) connects the corners of the distal tibial metaphysis. The angle between Line A and Line B (Angle 1) defines proximal tibial angulation, while the angle between Line A and Line C (Angle 2) defines the distal tibial angulation.

\section{Characterization of medial tibial curvature}

Two additional lines were drawn (Fig. 2). The first (Line D) is parallel to Line A and is placed tangential to the medial tibial
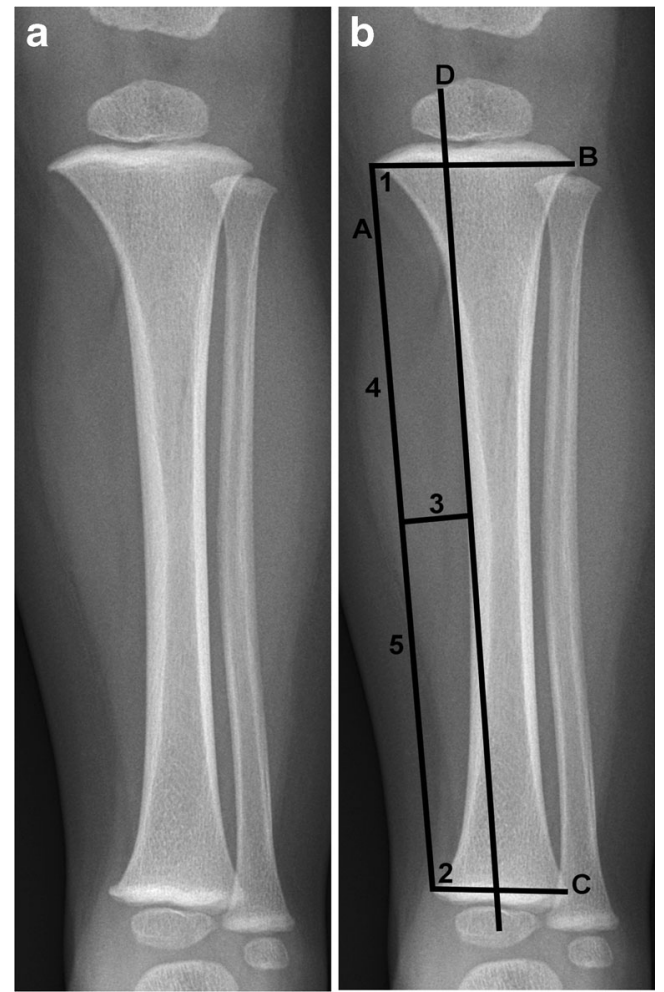

Fig. 2 Anteroposterior view of a left lower extremity without (a) and with measurements (b). Line A is the distance from the proximal corner of the metaphysis of the tibia to the distal corner of the tibial metaphysis on the medial aspect. Line B connects the medial and lateral corner of the proximal metaphysis. Line $\mathrm{C}$ connects the medial and lateral corner of the distal metaphysis. Line D is a tangent to the apex of the curved tibia and runs parallel to line A. Three is the distance between line A and D. Distances 4 and 5 are defined by the deepest point of the curvature of the tibia. One is the angle between Line A and B and 2 is the angle between line $\mathrm{A}$ and $\mathrm{C}$ cortex. An additional line (Line 3) measures the distance between Lines A and D and is placed at the deepest aspect of the tibial cortex. Line A is subdivided into segments 4 and 5 and was measured, as determined by the intersection of Line A and Line 3, representing the proximal and distal measures to the deepest aspect of the tibial curvature. The medial tibial curvature was characterized by two calculations: depth of curvature (Line 3 / Line A), and location of curvature (Line 4 / Line A).

\section{Measurements: lateral view}

\section{Posterior tibial length and angulation}

Three lines were drawn (Fig. 3). The first (Line E) connects the posterior aspect of the proximal tibial metaphysis to the
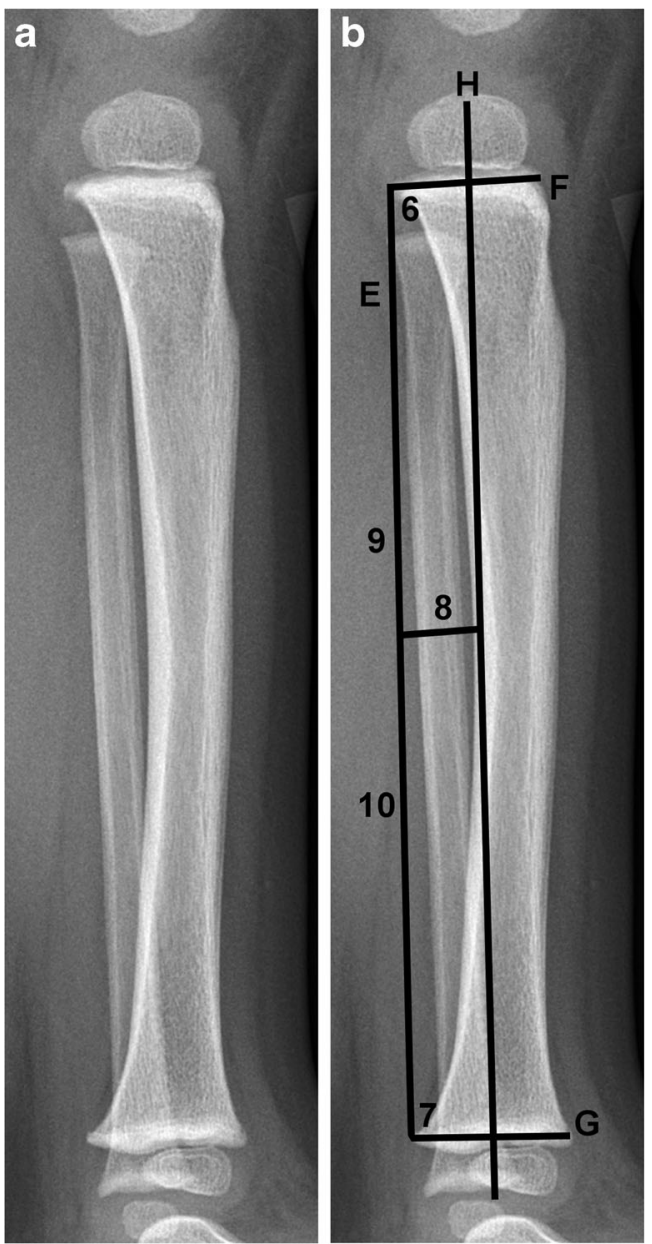

Fig. 3 Lateral view of a left lower extremity without (a) and with measurements $(\mathbf{b})$. Line $\mathrm{E}$ is the distance from the proximal corner of the metaphysis of the tibia to the distal corner of the tibial metaphysis on the medial aspect. Line F connects the medial and lateral corner of the proximal metaphysis. Line $\mathrm{G}$ connects the medial and lateral corner of the distal metaphysis. Line $\mathrm{H}$ is a tangent to the apex of the curved tibia and runs parallel to line E. Eight is the distance between line A and D. Distances 9 and 10 are defined by the deepest point of the curvature of the tibia. Six is the angle between Line A and B and 7 is the angle between line $\mathrm{A}$ and $\mathrm{C}$ 
Fig. 4 Bland Altman plot of a) Intrareader Agreement Reader 1, b) Intrareader Agreement Reader 2, c) Interreader Agreement

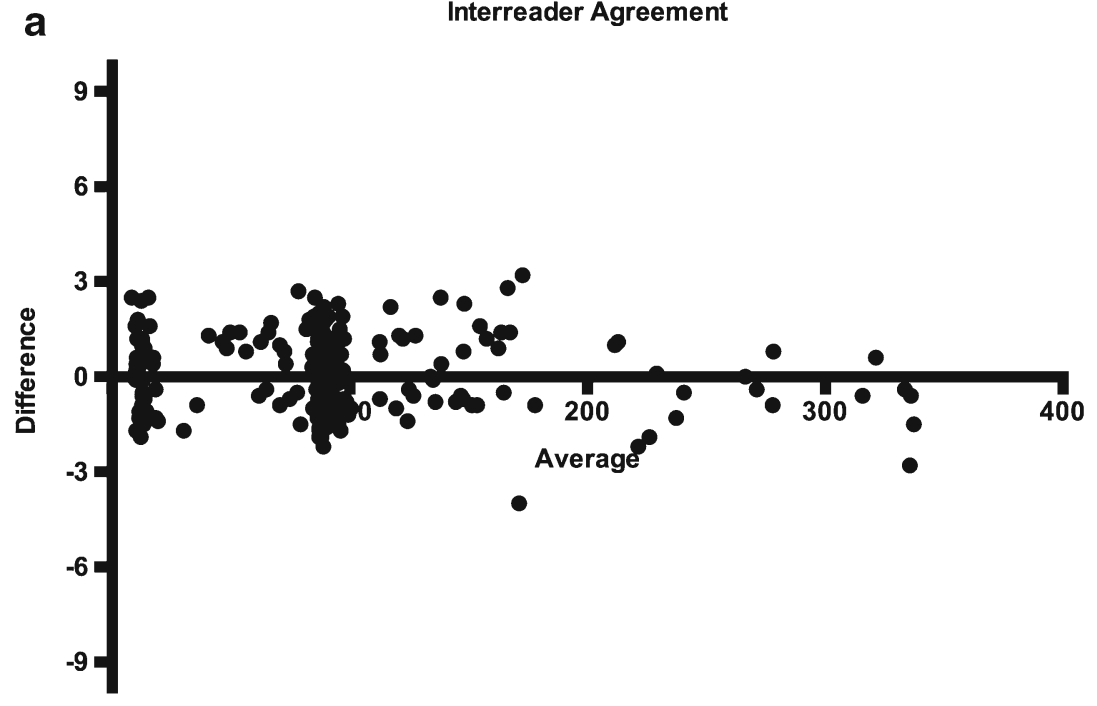

b

Intrareader Agreement Reader 1

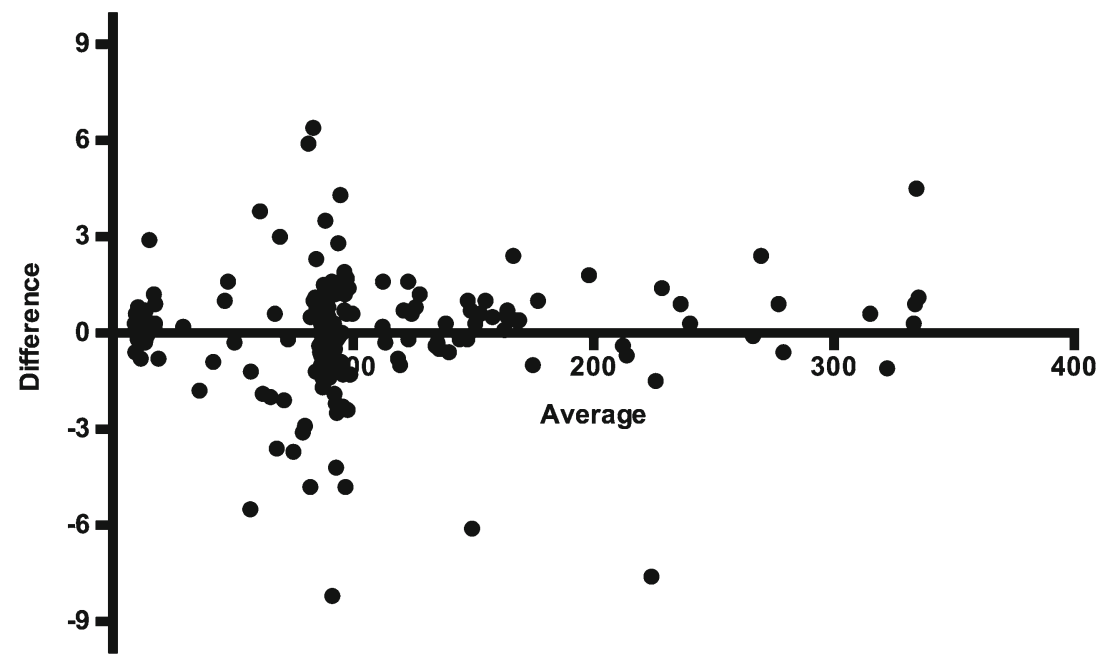

C

Intrareader Agreement Reader 2

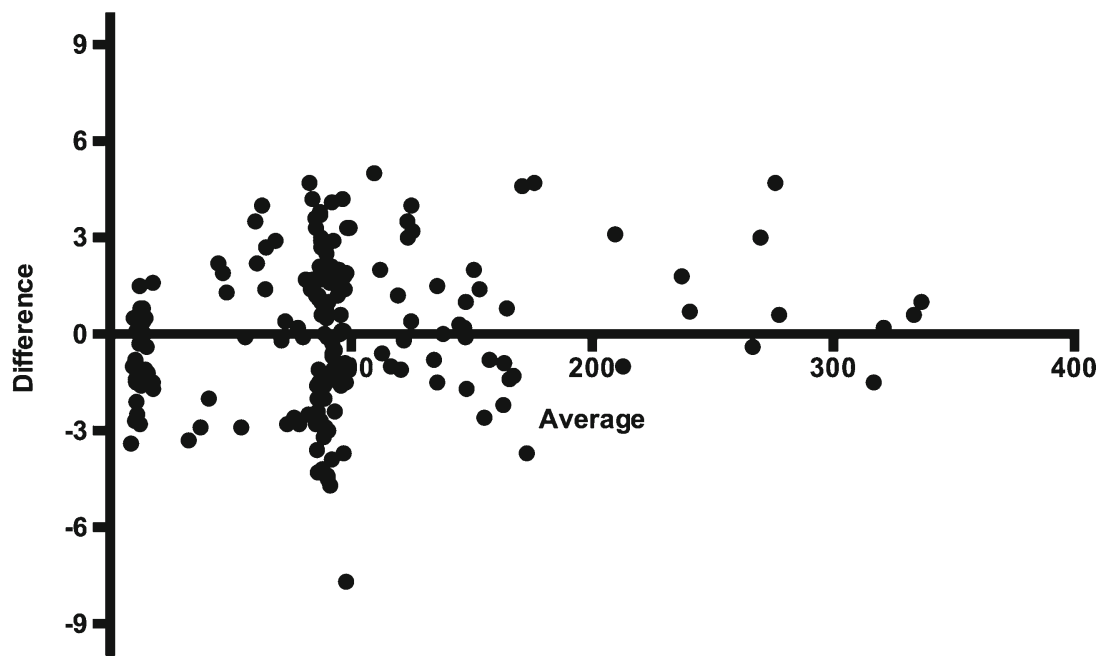


posterior aspect of the distal metaphysis, which defines the posterior tibial length. A second (Line F) connects the corners of the proximal tibial metaphysis, while a third line (Line G) connects the corners of the distal tibial metaphysis. The angle between Line E and Line F (Angle 6) defines proximal tibial angulation, while the angle between Line E and Line G (Angle 7) defines the distal tibial angulation.

\section{Characterization of posterior tibial curvature}

Two additional lines were drawn (Fig. 3). The first (Line H) is parallel to Line $\mathrm{E}$ and is placed tangential to the posterior tibial cortex. An additional line (Line 8) measures the distance between Lines $\mathrm{E}$ and $\mathrm{H}$ and is placed at the deepest aspect of the tibial cortex. Line $\mathrm{E}$ is subdivided into segments 9 and 10 and was measured, as determined by the intersection of Line $\mathrm{E}$ and Line 8, representing the proximal and distal measures to the deepest aspect of the tibial curvature. The posterior tibial curvature was characterized by two calculations: depth of curvature (Line 8 / Line E), and location of curvature (Line 9 / Line E).

\section{Statistical analysis}

The correlation of all measured angles and lines to age was calculated using a Pearson correlation. Ratios of Line 3/Line
Fig. 5 Linear regression of Angle 1 shows an increase with age. With (a) and without (b) data points. Continuous line represents the linear regression, the broken lines the cut off for normal values based on the $95 \%$ confidence interval
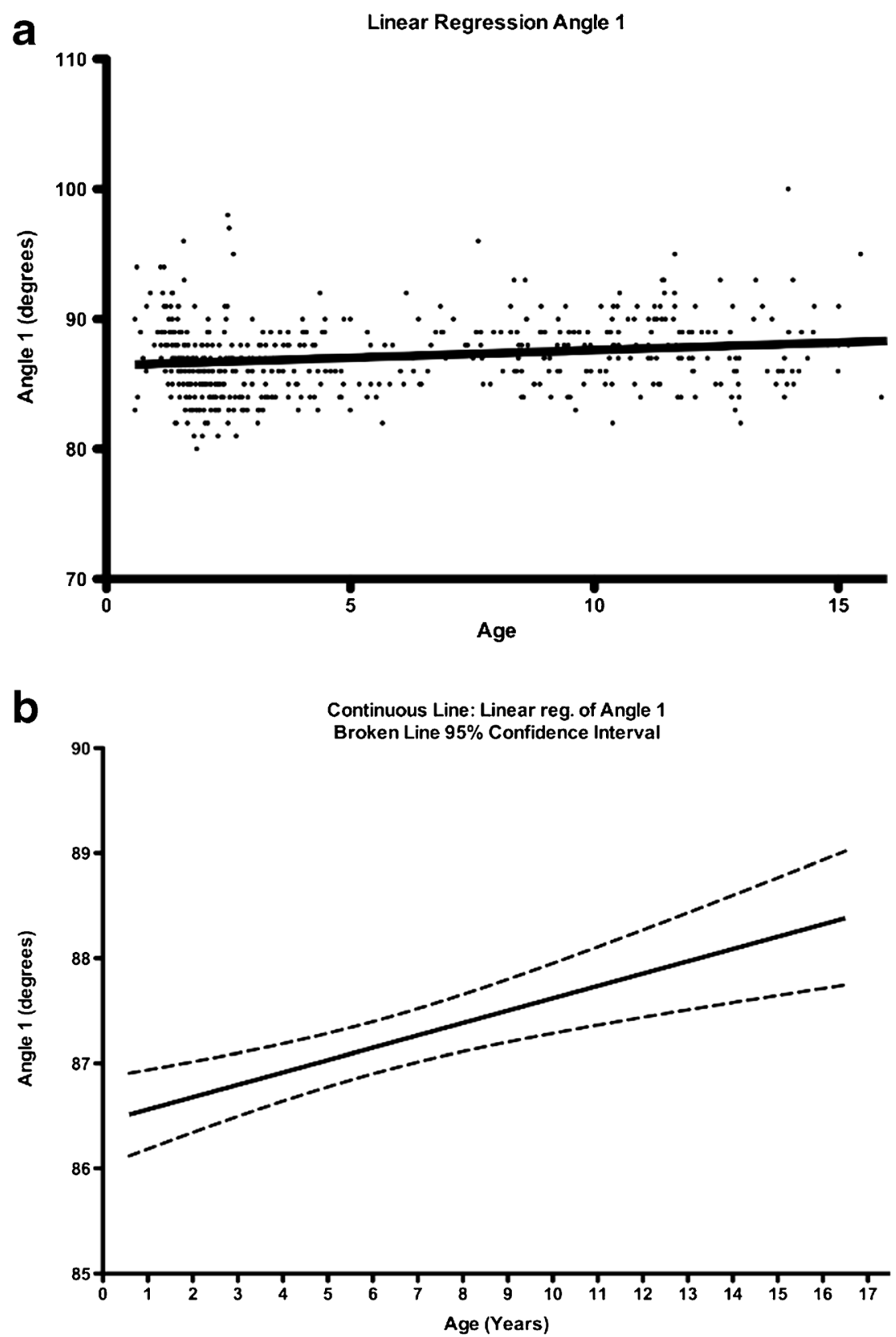
A, Line 4/Line A, Line 8/Line E, and Line 9/Line E were also correlated with subject age. Normal values with $95 \%$ confident interval (95\% of all measurements within upper and lower limits) related to age are calculated with linear regression. Intra- and inter-reader agreement was tested by using the Bland Altman Plot. The $P$ values of less than 0.05 were considered to indicate a significant difference. No correlation was considered for $r$-values $0-$ $0.09 /-0.09-0$, small $0.1-0.29 /-0.29--0.1$, medium $0.3-0.49 /-0.49--0.3$ and high for $0.5-1 /-1--0.5$. All analyses were performed by using a statistics program (Graph Pad Prism version 6.0d for Mac; GraphPad Software, San Diego, CA; www.graphpad.com).

\section{Results}

\section{Subject demographics}

A search of the Radiology Information System over 6 years revealed 4227 patients who had radiographs of a distal lower leg. After applying exclusion criteria, the final study group consisted of 526 subjects, where $55.5 \%(292 / 526)$ were male (average age: 6.363 years, range: $0.05-16.66$ years, standard deviation (SD): 4.7) and 44.5\% (234/526) were female (average age: 6.53 years, range: $0.58-16.78$ years, SD: 4.8).
Fig. 6 Linear regression of Angle 2 shows a decrease with age. With (a) and without (b) data points. Continuous line represents the linear regression, the broken lines the cut off for normal values based on the $95 \%$ confidence interval
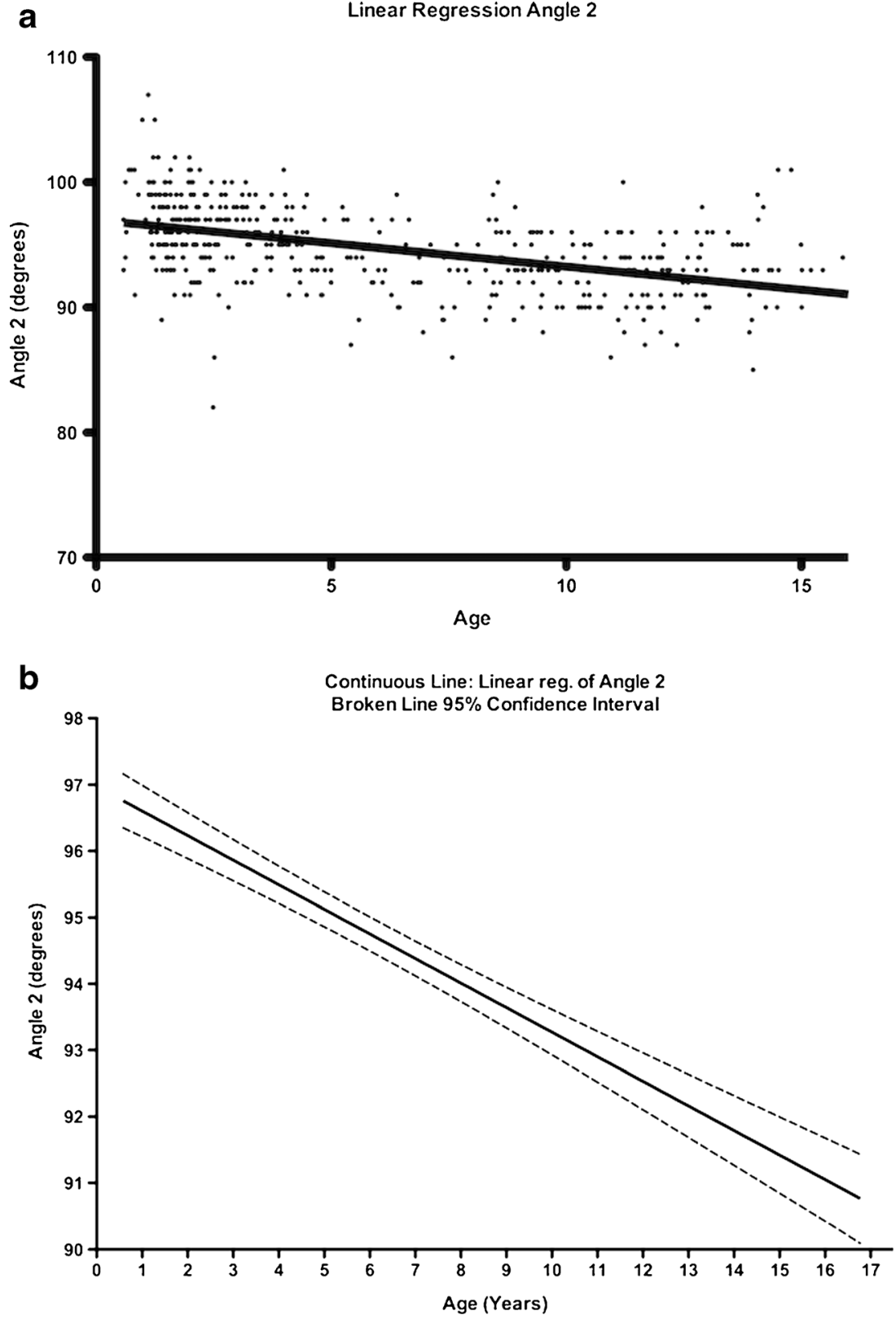


\section{Intra- and inter-reader agreement}

For Intrareader Agreement for Reader 1 the Bland Altman Plot (difference versus average) showed a bias of 0.1165 , standard deviation (SD) of bias was 1.938 and $95 \%$ limits of agreement from -3.915 to 3.682 (Fig. 4a). For Reader 2 it was -0.0106 , SD was 2.406 and limits of agreement -4.726 to 4.705 , respectively (Fig. 4b). For interreader agreement, the bias was 0.2050 , SD of bias was 1.595 and limits of agreement -2.921 to 3.331 (Fig. 4c). Since we detected no significant bias and good $95 \%$ limits of agreement, measurements of the whole study population were completed in consensus.

\section{Measurements: tibial angles}

The proximal tibial angle in anteroposterior view (Angle 1) ranged from 80 to 100 degrees (average 87.2, median 87,0, standard deviation 2.9). Linear regression in Fig. 5 shows an increase with age from 86.5 degrees at the age of 0 to 88 degrees at the age of 16 . The distal tibial angle in anteroposterior view (Angle 2) ranged from 82 to 107 (average 94.7, median 95.0, standard deviation 3.3). Linear regression in Fig. 6 shows a decrease with age from 96.8 degrees at the age of 0 to 90.5 degrees at the age of 16 . The proximal tibial angle in lateral view (Angle 6) ranged from 81 to 107 degrees (average 94.8, median 95.0, standard deviation 4.4). Linear regression in Fig. 7 shows an increase with age from 93
Fig. 7 Linear regression of Angle 6 shows an increase with age. With (a) and without (b) data points. Continuous line represents the linear regression, the broken lines the cut off for normal values based on the $95 \%$ confidence interval
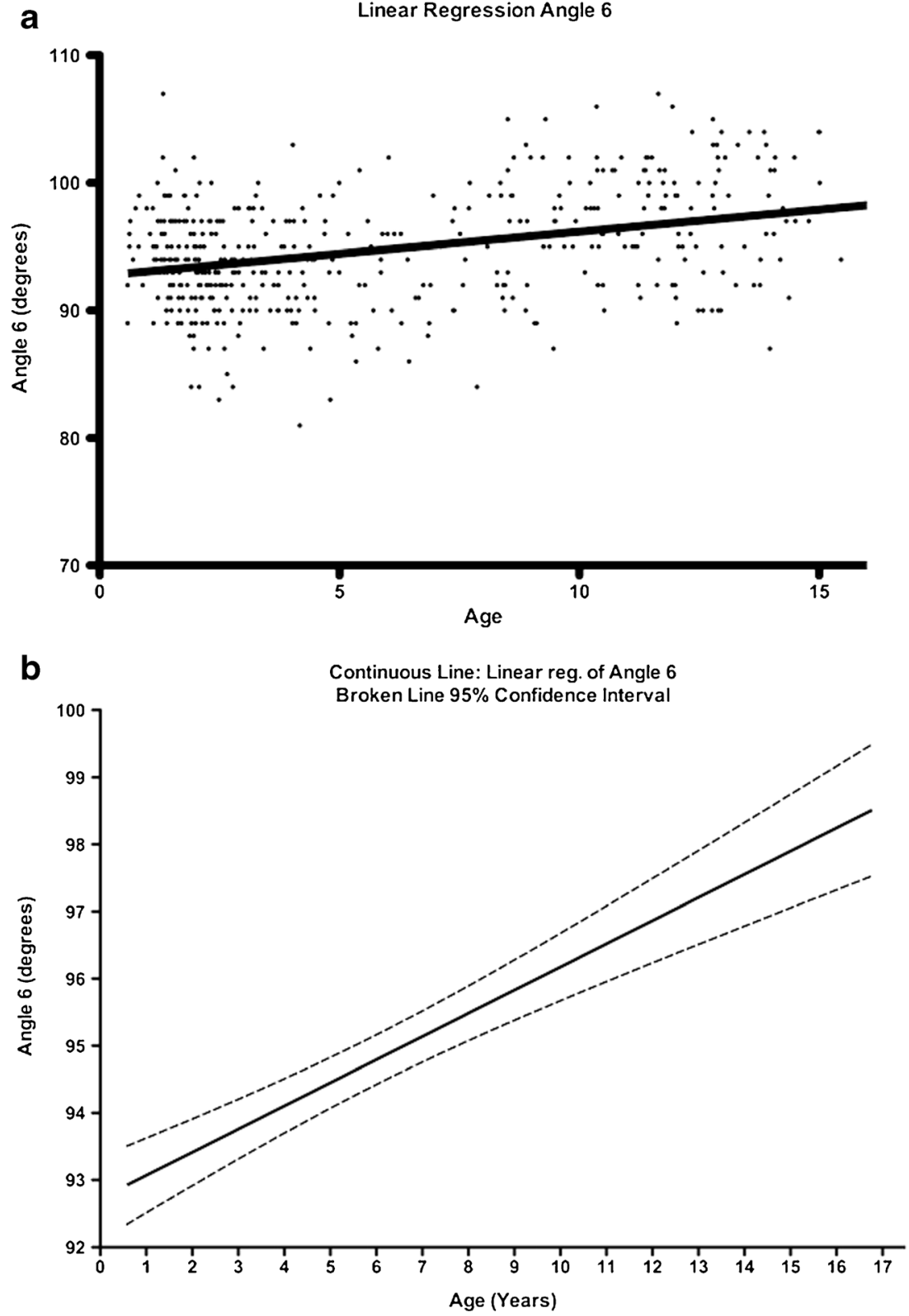
Fig. 8 Linear regression of Angle 7 shows a decrease with age with age. With (a) and without (b) data points. Continuous line represents the linear regression, the broken lines the cut off for normal values based on the $95 \%$ confidence interval
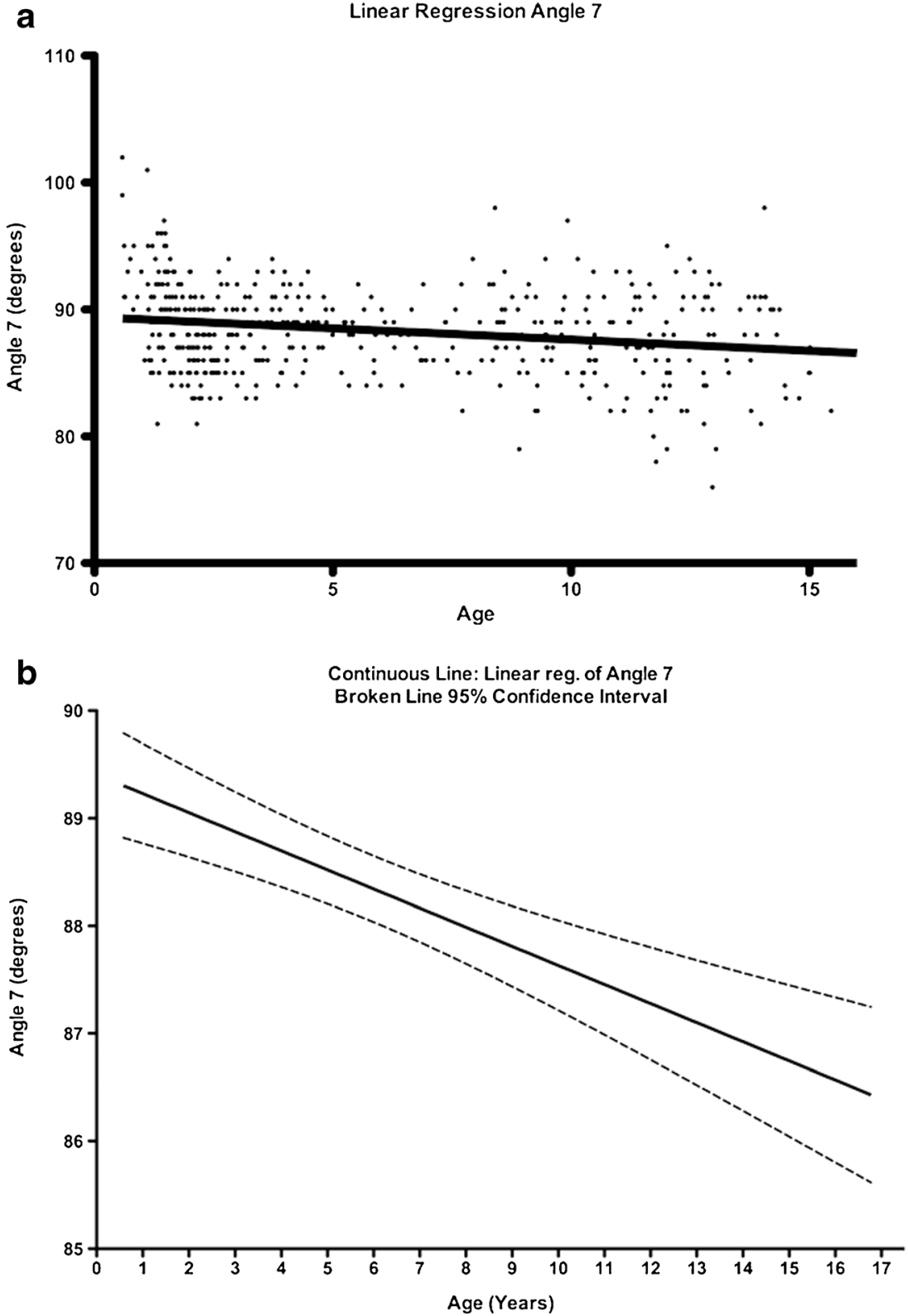

degrees at the age of 0 to 98 degrees at the age of 16 . The distal tibial angle in lateral view (Angle 7) ranged from $76-102$ degrees (average 88.4, median 88.0, standard deviation 3.5). Linear regression in Fig. 8 shows a decrease with age from

Table 1 Showing measurements with minimum, maximum, average, median values observed. Standard deviation of each measurement is provided

\begin{tabular}{|c|c|c|c|c|c|}
\hline Measurements & Minimum & Maximum & Average & Median & Standard Deviation \\
\hline Proximal tibial Angle in ap view (degree) & 80 & 100 & 87.2 & 87 & 2.9 \\
\hline Distal tibial Angle in ap view (degree) & 82 & 107 & 94.7 & 95 & 3.3 \\
\hline Proximal tibial Angle in lat view (degree) & 81 & 107 & 94.8 & 95 & 4.4 \\
\hline Distal tibial Angle in lat view (degree) & 76 & 102 & 88.4 & 88 & 3.5 \\
\hline Depth of curve in ap view (\%) & 0 & 11 & 6.6 & 6 & 1.7 \\
\hline Depth of curve in lat view (\%) & 2 & 13 & 6.8 & 7 & 1.6 \\
\hline Distance Deepest Point to Proximal metaphyseal corner (\%) in ap view & 14 & 77 & 44 & 45 & 16.6 \\
\hline Distance Deepest Point to Proximal metaphyseal corner (\%) in lat view & 4 & 47 & 15 & 15 & 15.1 \\
\hline
\end{tabular}


89.5 degrees at the age of 0 to 86.5 degrees at the age of 16 (Table 1).

\section{Measurements: curve characterization}

The depth of the tibial curve was calculated as percentages of distance between proximal and distal metaphyseal corners. Percentages of depth of curve in the anteroposterior view ranged from $0 \%$ to $11 \%$ (average $6.6 \%$, median $6.0 \%$, standard deviation $1.7 \%$ ). Linear regression in Fig. 9 shows a decrease with age from $8 \%$ at the age of 0 to $3.5 \%$ degrees at the age of 16 . Percentages of depth of curve in the lateral view ranged from $2 \%$ to $13 \%$ (average $6.8 \%$, median $7.0 \%$, standard deviation $1.6 \%$ ). Linear regression in Fig. 10 shows a decrease with age from $8.5 \%$ at the age of 0 to $3.5 \%$ at the age of 16. Percentages of distance of point of maximal depth to proximal metaphyseal corner to distance between proximal and distal metaphyseal corner were calculated. Percentages of distances of deepest point to proximal metaphyseal corner in anteroposterior view were $14 \%$ to $77 \%$ (average 44.0 , median 45 , standard deviation 16.6). Linear regression in Fig. 11 shows an increase with age from $37 \%$ at the age of 0 to $56 \%$ at the age of 16 . Percentages of distances of deepest point to proximal metaphyseal corner in lateral view were $4 \%$ to $47 \%$ (average 15.1, median 15, standard deviation 15.1). Linear regression in Fig. 12 shows a decrease with age from $16.5 \%$ at the age of 0 to $13 \%$ at the age of 16 (Table 1 ).
Fig. 9 Linear regression of depth of tibial curvature in anteroposterior view shows a decrease with age. Depth of Curve (distance 3 ) was calculated as percentage of Distance A. With (a) and without (b) data points. Continuous line represents the linear regression, the broken lines the cut off for normal values based on the $95 \%$ confidence interval
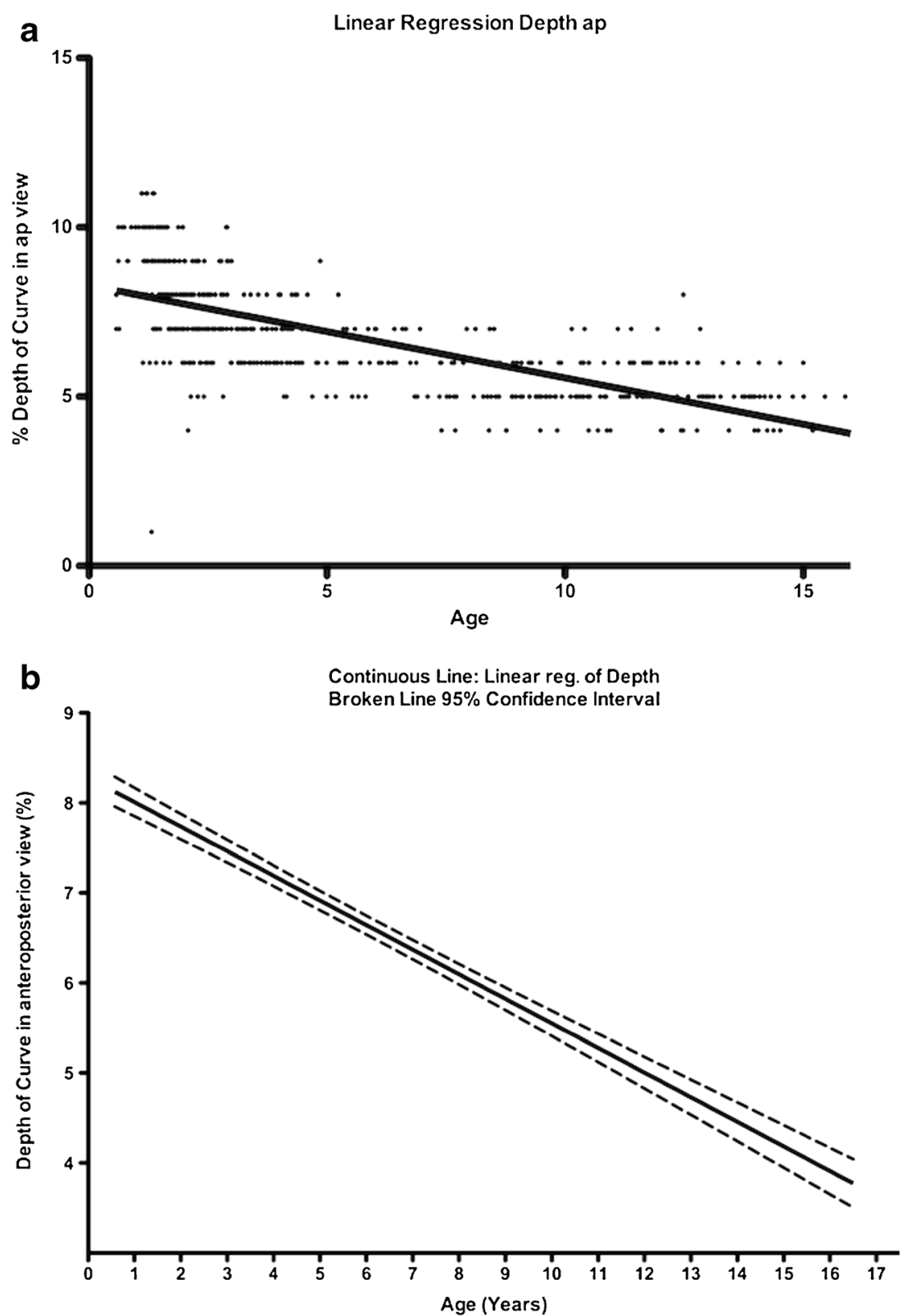
Fig. 10 Linear regression of depth of tibial curvature in lateral view shows a decrease with age. Depth of Curve (distance 8) was calculated as percentage of Distance E. With (a) and without (b) data points. Continuous line represents the linear regression, the broken lines the cut off for normal values based on the $95 \%$ confidence interval
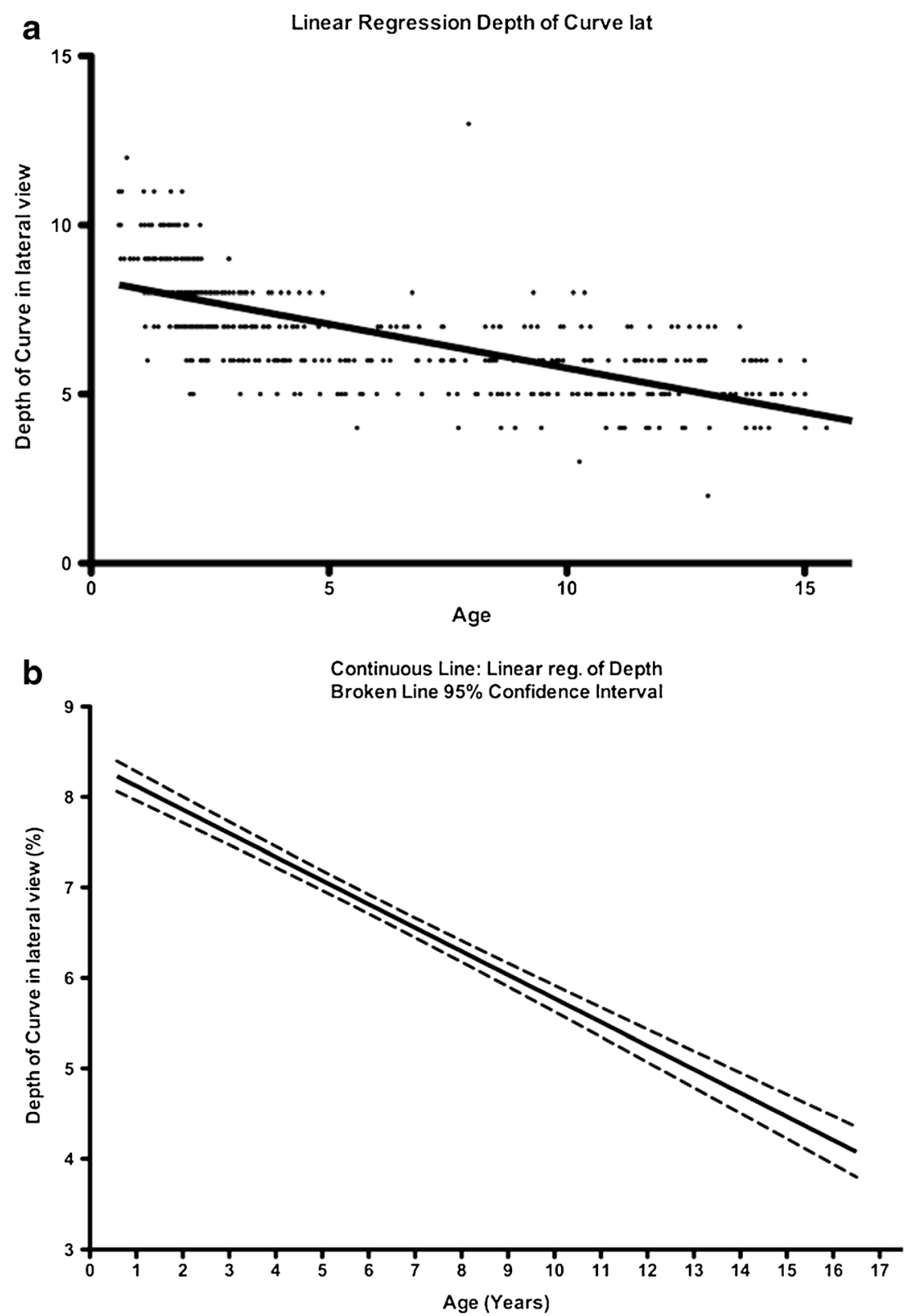

\section{Discussion}

Physiological bowing of the lower extremities, especially the tibia with varus angulation is normal in neonates and infants [1]. Such bowing is the result of the embryonal position with knee flexion and outer rotation of the knee joint [23]. Nevertheless, varus angulation is a frequent cause of referrals to paediatric orthopaedics [1]. There are numerous causes for bowing deformities of the tibia [1-9] and radiographs of the lower extremity are often required to determine whether the bowing should be considered physiologic or normal [19-21]. The results of the research define osseous landmarks on tibia radiographs to establish age-related normal values characterizing the physiological tibial bowing in children.

In our experience existing methods of measurements requiring weight bearing whole leg films is not practicable in children who cannot stand. Moreover measurements with use of the long axis of the tibia in "bowed legs" can be challenging or do not describe the location of bowing. In this study we present a measurement system characterizing physiological bowing with regard to degree, orientation, and localization with easy to define landmarks on plain films of the distal lower extremity and provide normal values for patients aged 0-16. Moreover, whole leg imaging applies higher radiation dose to patients. 
Fig. 11 Linear regression of distances of deepest point to proximal metaphyseal corner in ap view shows an increase with age. Distance (distance 4) was calculated as percentage of Distance A. With (a) and without (b) data points. Continuous line represents the linear regression, the broken lines the cut off for normal values based on the $95 \%$ confidence interval
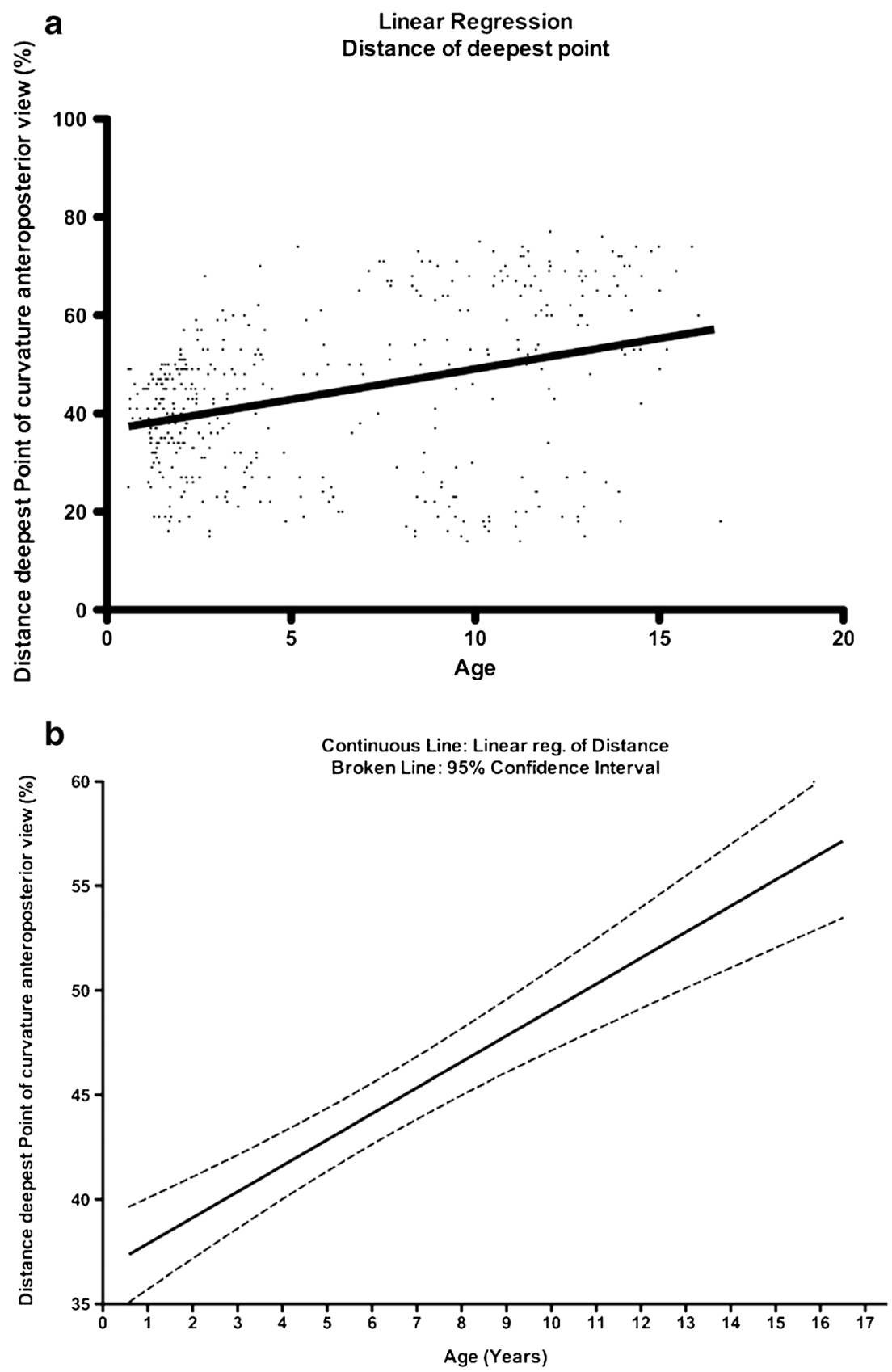

In our study we chose landmarks of the tibia that could readily be defined (Figs. 1,2). With regard to tibial angles, linear regression with $95 \%$ confidence interval band was within 2 degrees in all ages in both anteroposterior and lateral views, which should allow a confident assessment of abnormal angles. Hence we consider the measurements of these angles to be the most useful parameters. The age dependent approximation of angles to 90 degrees in anteroposterior views is compliant with physiological straightening of the tibia as described in prior studies [19, 20, 24]. Of interest , we did not observe the same for lateral views, as the angles increased with age. One possible explanation could be that in adolescents and adults the tibial plateau is tilted posteriorly [24, 25], and the distal tibial joint surface is tilted anteriorly [26].

With regard to characterization of tibial bowing, we consider assessment of depth of tibial bowing on anteroposterior radiographs in percentages as another useful parameter since the $95 \%$ confidence interval was less than $2 \%$. However, we do not consider the assessment of the deepest point of the curvature of the tibia useful when measured on the anteroposterior and lateral radiograph. Here the $95 \%$ confident interval was up to $10 \%$ in anteroposterior views and with 
Fig. 12 Linear regression of distances of deepest point to proximal metaphyseal corner in lateral view shows a decrease with age. Distance (distance 9) was calculated as percentage of distance E. With (a) and without (b) data points. Continuous line represents the linear regression, the broken lines the cut off for normal values based on the $95 \%$ confidence interval
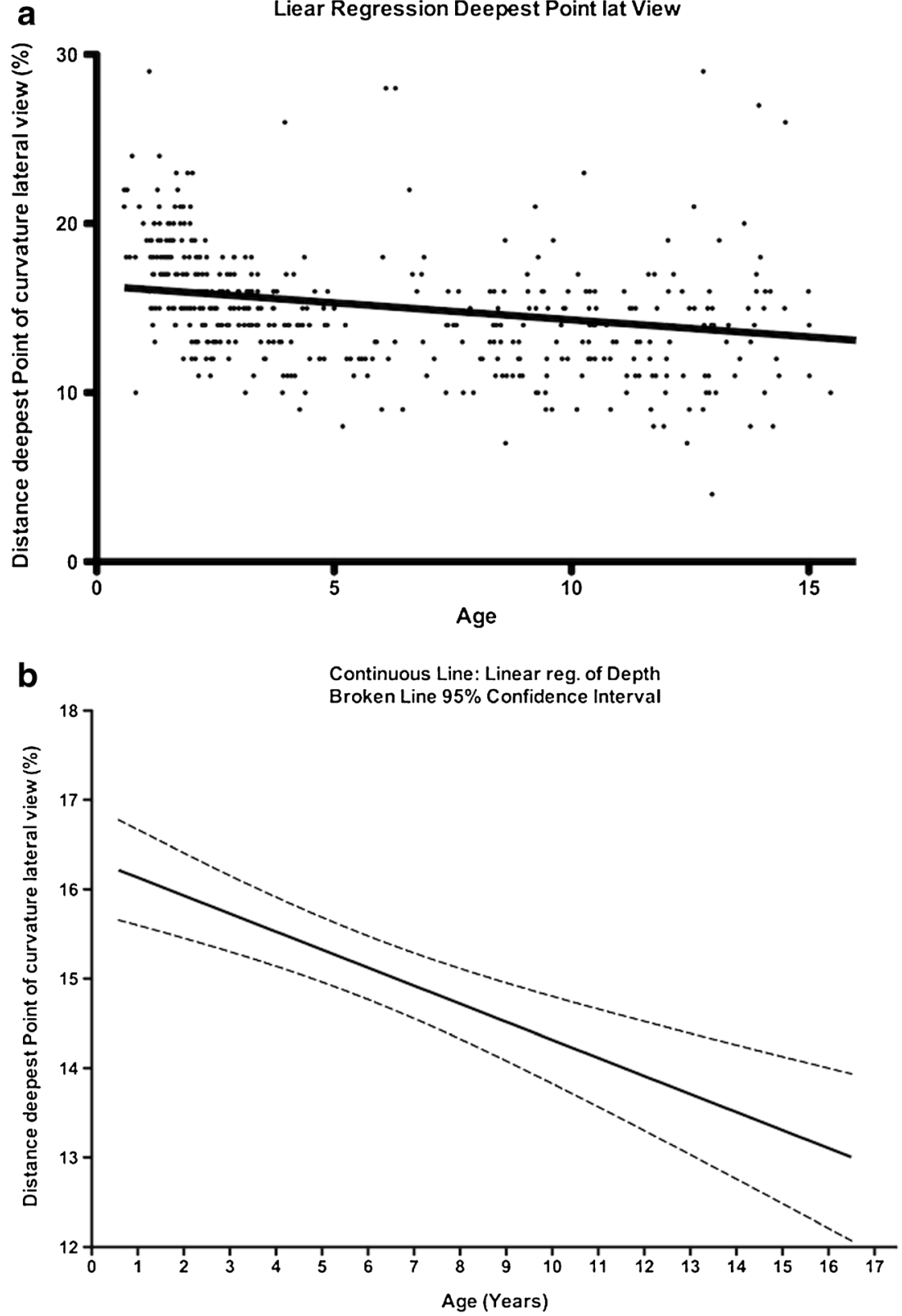

up to $5 \%$, slightly better in lateral views. This can be explained by difficulties defining this point in "straight" tibiae with increasing age.

We acknowledge several limitations of this study. One limitation of our study is its retrospective design. Another limitation is a selection bias given the fact that all our subjects had undergone radiographs due to symptoms in the lower leg; however, it would be extremely difficult to justify a true cross sectional study that would include an invasive study such as plain film of asymptomatic individuals in a paediatric patient group. In order to reduce selection bias, we excluded patients with clinicallysuspected abnormal bone development or radiographic abnormalities not related to bowing.
In conclusion, age-dependent physiological tibial varus angulation can be assessed with the measurement system presented in this study. The narrow bands of $95 \%$ confidence interval also allow the assumption that abnormal tibial bowing is present with differences of angles above 2 degrees and percentages compared to normal values provided in this study.

Acknowledgments The scientific guarantor of this publication is Olaf Magerkurth. The authors of this manuscript declare no relationships with any companies, whose products or services may be related to the subject matter of the article. This study has received funding by Pro UKBB Foundation. No complex statistical methods were necessary for this paper. Institutional Review Board approval was obtained. Written informed consent was waived by the Institutional Review Board. Methodology: retrospective, cross sectional study, performed at one institution. 


\section{References}

1. Lovell WW, Winter RB, Morrissy RT, Weinstein SLV (eds) (1996) Lovell and Winter's pediatric orthopaedics, 4th edn. LippincottRaven, Philadelphia, PA, pp 1047-1075

2. Caffey J (1947) Prenatal bowing and thickening of tubular bones, with multiple cutaneous dimples in arms and legs; a congenital syndrome of mechanical origin. Am J Dis Child 74(5):543-562, 1911

3. Hofmann A, Wenger DR (1981) Posteromedial bowing of the tibia. Progression of discrepancy in leg lengths. J Bone Joint Surg Am 63(3):384-388

4. Hefti F, Bollini G, Dungl P, Fixsen J, Grill F, Ippolito E et al (2000) Congenital pseudarthrosis of the tibia: history, etiology, classification, and epidemiologic data. J Pediatr Orthop B 9(1):11-15

5. Lovell WW, Winter RB, Morrissy RT, Weinstein SLV (eds) (1996) Lovell and Winter's pediatric orthopaedics, 4th edn. LippincottRaven, Philadelphia, PA, pp 305-344

6. Ablin DS (1998) Osteogenesis imperfecta: a review. Can Assoc Radiol J J Assoc Can Radiol 49(2):110-123

7. Lovell WW, Winter RB, Morrissy RT, Weinstein SLV (eds) (1996) Lovell and Winter's pediatric orthopaedics, 4th edn. LippincottRaven, Philadelphia, PA, pp 137-201

8. Schmickel RD, Heidelberger KP, Poznanski AK (1973) The campomelique syndrome. J Pediatr 82(2):299-302

9. Jackson DW, Cozen L (1971) Genu valgum as a complication of proximal tibial metaphyseal fractures in children. J Bone Joint Surg Am 53(8):1571-1578

10. Raney EM, Topoleski TA, Yaghoubian R, Guidera KJ, Marshall JG (1998) Orthotic treatment of infantile tibia vara. J Pediatr Orthop 18(5):670-674

11. Zionts LE, Shean CJ (1998) Brace treatment of early infantile tibia vara. J Pediatr Orthop 18(1):102-109

12. McCarthy JJ, MacIntyre NR 3rd, Hooks B, Davidson RS (2009) Double osteotomy for the treatment of severe Blount disease. J Pediatr Orthop 29(2):115-119

13. Masrouha KZ, Sraj S, Lakkis S, Saghieh S (2011) High tibial osteotomy in young adults with constitutional tibia vara. Knee Surg Sports Traumatol Arthrosc Off J ESSKA 19(1):89-93

14. Putzeys P, Wilmes P, Merle M (2013) Triple tibial osteotomy for the correction of severe bilateral varus deformity in a patient with late- onset Blount's disease. Knee Surg Sports Traumatol Arthrosc Off J ESSKA 21(3):731-735

15. Clarke SE, McCarthy JJ, Davidson RS (2009) Treatment of Blount disease: a comparison between the multiaxial correction system and other external fixators. J Pediatr Orthop 29(2):103-109

16. Bito H, Takeuchi R, Kumagai K, Aratake M, Saito I, Hayashi R et al (2009) A predictive factor for acquiring an ideal lower limb realignment after opening-wedge high tibial osteotomy. Knee Surg Sports Traumatol Arthrosc Off J ESSKA 17(4):382-389

17. Saragaglia D, Nemer C, Colle P-E (2008) Computer-assisted double level osteotomy for severe genu varum. Sports Med Arthrosc Rev 16(2):91-96

18. Kodkani PS (2007) Dome osteotomy of the proximal tibia for genu varum treated with a new fixation device. J Knee Surg 20(2):111119

19. Paley D, Tetsworth K (1992) Mechanical axis deviation of the lower limbs. Preoperative planning of multiapical frontal plane angular and bowing deformities of the femur and tibia. Clin Orthop 280:6571

20. Paley D, Tetsworth K (1992) Mechanical axis deviation of the lower limbs. Preoperative planning of uniapical angular deformities of the tibia or femur. Clin Orthop 280:48-64

21. Levine AM, Drennan JC (1982) Physiological bowing and tibia vara. The metaphyseal-diaphyseal angle in the measurement of bowleg deformities. J Bone Joint Surg Am 64(8):1158-1163

22. Paley D (2002) Principles of deformity correction. Springer, Berlin; New York, $806 \mathrm{p}$

23. Cheema JI, Grissom LE, Harcke HT (2003) Radiographic Characteristics of Lower-Extremity Bowing in Children1. RadioGraphics 23(4):871-880

24. Salenius P, Vankka E (1975) The development of the tibiofemoral angle in children. J Bone Joint Surg Am 57(2):259-261

25. Takatsu T, Itokazu M, Shimizu K, Brown TD (1998) The function of posterior tilt of the tibial component following posterior cruciate ligament-retaining total knee arthroplasty. Bull Hosp Jt Dis N Y N 57(4):195-201

26. Magerkurth O, Knupp M, Ledermann H, Hintermann B (2006) Evaluation of hindfoot dimensions: a radiological study. Foot Ankle Int Am Orthop Foot Ankle Soc Swiss Foot Ankle Soc 27(8):612-616 